\title{
Combined Effects of Thermal Diffusion and Diffusion-Thermo Effects on Transient MHD Natural Convection and Mass Transfer Flow in a Vertical Channel with Thermal Radiation
}

\author{
Isah Bala Yabo1, Basant Kumar Jha², Jeng-Eng Lin ${ }^{3}$ \\ ${ }^{1}$ Department of Mathematics, Usmanu Danfodiyo University, Sokoto, Nigeria \\ ${ }^{2}$ Department of Mathematics, Ahmadu Bello University, Zaria, Nigeria \\ ${ }^{3}$ Department of Mathematical Sciences, George Mason University, Virginia, USA \\ Email: isahby1973@gmail.com, isah.yabo@udusok.edu.ng, basant777@yahoo.co.uk, jelin@gmu.edu
}

How to cite this paper: Yabo, I.B., Jha, B.K. and Lin, J.-E. (2016) Combined Effects of Thermal Diffusion and Diffusion-Thermo Effects on Transient MHD Natural Convection and Mass Transfer Flow in a Vertical Channel with Thermal Radiation. Applied Mathematics, 7, 2354-2373.

http://dx.doi.org/10.4236/am.2016.718185

Received: October 15, 2016

Accepted: December 19, 2016

Published: December 22, 2016

Copyright $\odot 2016$ by authors and Scientific Research Publishing Inc. This work is licensed under the Creative Commons Attribution International License (CC BY 4.0). http://creativecommons.org/licenses/by/4.0/ (c) (i) Open Access

\begin{abstract}
We study the nonlinear coupled evolution equations which model the transient MHD natural convection and mass transfer flow of viscous, incompressible and electrically conducting fluid between two infinite vertical plates in the presence of the transversal magnetic field, thermal radiation, thermal diffusion and diffusion-thermo effects. Both analytical and numerical methods are used for this study.
\end{abstract}

\section{Keywords}

Transient MHD, Diffusion-Thermo, Thermal Diffusion

\section{Introduction}

Heat and mass transfer flow of an electrically conducting fluid between parallel plates in the presence of a magnetic field and thermal radiation is of a special significance both naturally and in many industrial applications such as magnetohydrodynamic power generators, pumps, cooling of nuclear reactors, geothermal systems, thermal insulators, nuclear waste disposal, petroleum and polymer technology, heat exchangers and others [1]. One of the earliest studies in this field was carried out by [2]. They analyzed the influence of the effects of a transverse uniform magnetic field on the flow of a viscous incompressible electrically conducting fluid exiting through parallel stationary plates that are insulated. Since then, several aspects of this problem have been investigated [3]. 
It has been found that an energy flux can be generated not only by temperature gradients but also by concentration gradients. The heat transfer caused by concentration gradient is called the diffusion-thermo or Dufour effect while mass transfer caused by temperature gradients is called Soret or thermal diffusion effect. Thus, Soret effect is referred to species differentiation developing in an initial homogenous mixture submitted to a thermal gradient and the Dufour effect referred to the heat flux produced by a concentration gradient. Fouriers neglect these effects on the basis that they are of a smaller order of magnitude, than the effects described and Ficks laws in heat and mass transfer processes [4]. [5] found that the thermal diffusion effect assumes significance for instance in isotope separation and in mixtures between gases with very light molecular weight $\left(\mathrm{H}_{2}, \mathrm{He}\right)$ and for medium molecular weight $\left(\mathrm{N}_{2}\right.$, air), the diffusion-thermo effects constitute a substantial effect such that it cannot be ignored. Hence several models with Soret and Dufour effect in different heat and mass transfer problems have been studied. Recently, [6] considered Dufour effect on the free-convection and mass transfer flow in a vertical channel when the boundaries are subjected to symmetric concentration and thermal input. [7] presented a theoretical treatment of unsteady hydromagnetic flow and heat and mass transfer of an incompressible electrically-conducting and radiating fluid in a vertical filled with porous medium taking into account the Soret number. [4] considered the effect of thermo-diffusion on MHD mixed convective heat and mass transfer flow of a viscous fluid through a porous medium with radiation, heat generation and chemical reaction.

The role of thermal radiation on MHD flow and heat transfer problem continues to have great deal of interest. For instance at high operating temperature, radiation effect can be quite significant. For instance, in many processes in engineering areas such as Nuclear power plants, gas turbines and the various propulsion devices for aircraft, missiles, satellites and space vehicles, knowledge of thermal radiation effect becomes very important for the design of the pertinent equipment [8]. [9] explained the behaviors of chemically reacted unsteady MHD free convection flow in the presence of thermal diffusion and diffusion thermo. [10] analyzed Soret and Dufour effects on unsteady MHD mixed convection flow past a radiative vertical porous plate embedded in a porous medium with chemical reaction. [11] discussed the effect of Soret and Dufour number on an unsteady magnetohydrodynamic free convective fluid flow past a vertical porous plate in the presence of suction or injection. [9] studied chemical reaction effect on an unsteady MHD free convection flow past an infinite vertical accelerated plate with constant heat flux, thermal diffusion and diffusion thermo. [12] examined the thermal radiation effect on the MHD three-dimensional flow of Eyring-power fluid. More recently, [13] carried out a parametric study on radiation, Soret and Dufour effects in MHD channel flow bounded by a long wavy wall and a uniformly moving parallel at wall.

In the present article, we consider the thermal diffusion and diffusion-thermo effect on MHD free convection and mass transfer flow which, will be analysed by means of non-linear Rosseland diffusion approximation. 


\section{Mathematical Analysis}

Consider a two-dimensional transient combined free convective and mass transfer flow of a viscous, incompressible and electrically conduction fluid between two infinite vertical parallel plates. A uniform transverse magnetic field of magnitude $B_{0}$ is applied in the presence of an incident radiation flux of intensity $q_{r}$, which absorbed by the plate and transferred to the fluid as shown in Figure 1. At time $t \leq 0$, both the fluid and plates are assumed to be at rest at constant temperature $T_{0}$ and constant concentration $C_{0}$ respectively. At time $t>0$ the temperature of the plate situated at $y^{\prime}=0$ and concentration rise to $T_{w}$ and $C_{w}$ while the other plate at a distance $H$ from it, is fixed and maintained at temperature $T_{0}$. The stream wise coordinate is denoted by $x^{\prime}$ taken vertically upward direction and that normal to it is denoted by $y^{\prime}$. The flow is assumed laminar and fully developed means that the axial ( $x$-direction) velocity depends only on transverse coordinate, $y^{\prime}$. Therefore from the continuity equation, the transverse velocity, the variable $v$ is equated to zero. Since the plates are of infinite length, the velocity, temperature and concentration are function of $y^{\prime}$ and $t^{\prime}$ alone. Using the Boussinesq's approximation, the governing equations for the present physical situation in the dimensional form are:

$$
\begin{gathered}
\frac{\partial u^{\prime}}{\partial t^{\prime}}=v \frac{\partial^{2} u^{\prime}}{\partial y^{\prime 2}}+g \beta\left(T^{\prime}-T_{0}\right)+g \beta^{*}\left(C^{\prime}-C_{0}\right)-\frac{\sigma_{1} B_{0}^{2} u^{\prime}}{\rho} \\
\frac{\partial T^{\prime}}{\partial t^{\prime}}=\alpha\left[\frac{\partial^{2} T^{\prime}}{\partial y^{\prime 2}}-\frac{1}{K} \frac{\partial q_{r}}{\partial y^{\prime}}\right]+D_{m}\left[\frac{\partial^{2} C^{\prime}}{\partial y^{\prime 2}}\right] \\
\frac{\partial C^{\prime}}{\partial t^{\prime}}=D\left[\frac{\partial^{2} C^{\prime}}{\partial y^{\prime 2}}\right]+D_{t}\left[\frac{\partial^{2} T^{\prime}}{\partial y^{\prime 2}}\right]
\end{gathered}
$$

where $T_{0}$ is the initial temperature of the fluid and porous plates, $T^{\prime}$ is the dimensional temperature of the fluid, $\alpha$ is the thermal diffusivity, $K$ is the thermal

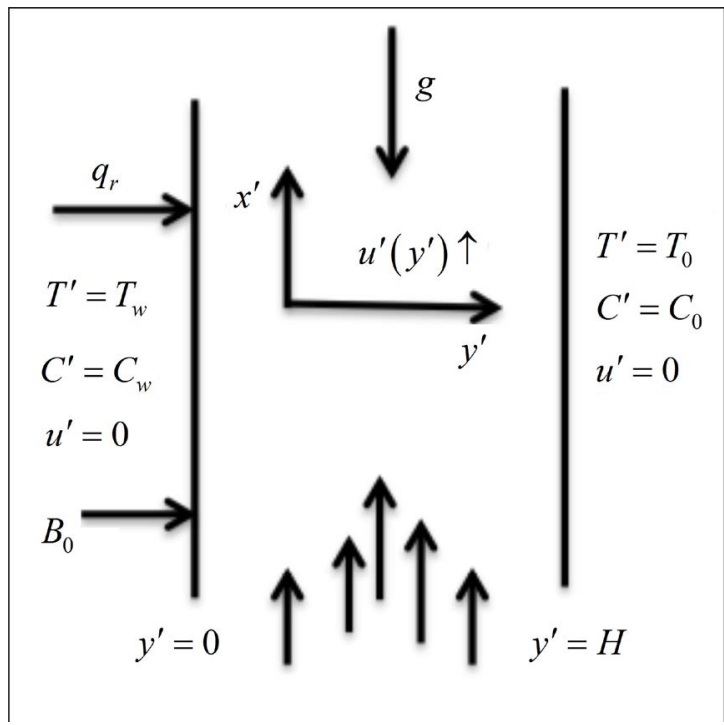

Figure 1. Schematic diagram of the problem. 
conductivity, $\rho$ is the density of the fluid, $\beta$ is the coefficient of the thermal expansion, $\sigma_{1}$ is the fluid electrical conductivity, $g$ is the gravitational acceleration and $B_{0}$ is the strength of applied magnetic field. The flow is assumed laminar and fully developed. Meaning that the axial ( $x$-direction) velocity depends only on transverse coordinate, $y^{\prime}$.

$$
q_{r}=-\frac{4 \sigma \partial T^{\prime 4}}{3 \kappa^{*} \partial y^{\prime}}
$$

The quantity $q_{r}$ appearing on the right hand side of Equation (2) represents the radiative heat flux in the $y^{\prime}$-direction where the radiative heat flux in the $x$-direction is considered insignificant in comparison with that in the $y^{\prime}$-direction. The radiative heat flux term in the problem is simplified by using the Rosseland diffusion approximation for an optically thick fluid according to [14], where $\sigma$ is Stefan-Boltzmann constant and $\kappa^{*}$ the mean absorption coefficient.

This approximation is valid for intensive absorption, that is, for an optically thick boundary layer. Despite these shortcomings, the Rosseland approximation has been used with success in a variety of problems ranging from the transport of radiation through gases at low density to the study of the effects of radiation on blast waves by nuclear explosion [14].

The required initial and boundary conditions to be satisfied are

$$
\begin{aligned}
& t^{\prime} \leq 0: \quad u^{\prime}=0 \quad T^{\prime}=T_{0} \quad \text { for } 0 \leq y^{\prime} \leq H \\
& t^{\prime}>0:\left\{\begin{array}{llll}
u^{\prime}=0 & T^{\prime}=T_{w} & \text { at } \quad y^{\prime}=0 \\
u^{\prime}=0 & T^{\prime}=T_{0} & \text { at } & y^{\prime}=H
\end{array}\right.
\end{aligned}
$$

To obtain the non-dimensional form of the above equations, the following dimensionless variables are introduced.

$$
\begin{aligned}
& t=\frac{t^{\prime} v}{H^{2}} ; y=\frac{y^{\prime}}{H} ; \operatorname{Pr}=\frac{v}{\alpha} ; R=\frac{4 \sigma\left(T_{w}-T_{0}\right)^{3}}{\kappa^{*} K} ; u=u^{\prime} v\left[g \beta H^{2}\left(T_{w}-T_{0}\right)\right]^{-1} \\
& \left.M^{2}=\frac{\sigma_{1} B_{0}^{2} H^{2}}{v \rho} ; C_{T}=\frac{T_{0}}{T_{w}-T_{0}} ; \theta=\frac{T^{\prime}-T_{0}}{T_{w}-T_{0}} ; \phi=\frac{C^{\prime}-C_{0}}{C_{w}-C_{0}} ; N=\frac{\beta^{*}\left(C_{w}-C_{0}\right)}{\beta\left(T_{w}-T_{0}\right)}\right\} \\
& S_{t}=\frac{D_{t}\left(T_{w}-T_{0}\right)}{D_{m}\left(C_{w}-C_{0}\right)} ; c=\frac{v}{D_{m}} ; D_{f}=\frac{D_{c}\left(C_{w}-C_{0}\right)}{\alpha\left(T_{w}-T_{0}\right)}
\end{aligned}
$$

Using Equations ((4) and (6)) in Equations (1)-(3), we obtain the following dimensionless equations for velocity, temperature and concentration respectively.

$$
\begin{gathered}
\frac{\partial u}{\partial t}=\frac{\partial^{2} u}{\partial y^{2}}+[\theta+N \phi]-M^{2} u \\
\operatorname{Pr}\left[\frac{\partial \theta}{\partial t}\right]=\left[1+\frac{4}{3} R\left(C_{T}+\theta\right)^{3}\right]\left(\frac{\partial^{2} \theta}{\partial y^{2}}\right)+4 R\left[C_{T}+\theta\right]^{2}\left(\frac{\partial \theta}{\partial y}\right)^{2} D_{f}\left[\frac{\partial^{2} \phi}{\partial y^{2}}\right] \\
S c\left[\frac{\partial \phi}{\partial t}\right]=\frac{\partial^{2} \phi}{\partial y^{2}}+S_{t}\left[\frac{\partial^{2} \theta}{\partial y^{2}}\right] \\
t \leq 0: u=0 ; \theta=0 ; \phi=0 ; \text { for } 0 \leq y \leq 1
\end{gathered}
$$




$$
t>0:\left\{\begin{array}{l}
u=0 ; \theta=1 ; \phi=1 ; \text { at } y=0 \\
u=0 ; \theta=0 ; \phi=0 ; \text { at } y=1
\end{array}\right.
$$

\section{Analytical Solution}

The governing equations presented in the previous section are highly nonlinear and exhibited no exact solutions. In general such solution can be very useful in validating computer routines of complicated time dependent two or three-dimensional free convective and radiating conducting fluid and comparison with experimental data. It is therefore of interest to reduce the governing equations of the present problem to the form that can be solved analytically. A special case of the present problem that exhibit analytical solution is the problem of steady state MHD natural convection flow in a vertical channel in a presence of thermal radiation, thermal diffusion and diffusion-thermo effects. The resulting steady state equations and boundary conditions for this special case can be written as

$$
\begin{gathered}
\frac{\mathrm{d}^{2} u}{\mathrm{~d} y^{2}}-M^{2} u=-[\theta+N \phi] \\
{\left[1+\frac{4}{3} R\left(C_{T}+\theta\right)^{3}\right] \frac{\mathrm{d}^{2} \theta}{\mathrm{d} y^{2}}+4 R\left[C_{T}+\theta\right]^{2}\left(\frac{\mathrm{d} \theta}{\mathrm{d} y}\right)=-D_{f}\left[\frac{\mathrm{d}^{2} \phi}{\mathrm{d} y^{2}}\right]} \\
\frac{\mathrm{d}^{2} \phi}{\mathrm{d} y^{2}}=-S_{t}\left[\frac{\mathrm{d}^{2} \theta}{\mathrm{d} y^{2}}\right]
\end{gathered}
$$

the boundary conditions are

$$
\left\{\begin{array}{l}
u=0 ; \theta=1 ; \phi=1 ; \quad \text { at } \quad y=0 \\
u=0 ; \theta=0 ; \phi=0 ; \text { at } y=1
\end{array}\right.
$$

To construct analytical solutions of Equations ((11) to (13)) subject to (14), it is assumed that the radiation parameter is small and taking a power series expansion in the radiation parameter $R$ employs a regular perturbation method.

$$
\begin{aligned}
& u(y)=u_{0}+R u_{1}(y)+\cdots=\sum_{j=0}^{\infty} R^{j} u_{j}(y) \\
& \left.\theta(y)=\theta_{0}+R \theta_{1}(y)+\cdots=\sum_{j=0}^{\infty} R^{j} \theta_{j}(y)\right\} \\
& \phi(y)=\phi_{0}+R \phi_{1}(y)+\cdots=\sum_{j=0}^{\infty} R^{j} \phi_{j}(y)
\end{aligned}
$$

where $R$ is the radiation parameter $(R \ll 1)$. The second and higher order of terms of $R$ gives correction to $u_{0}, \theta_{0}$ and $\phi_{0}$ account for thermal radiation effects. Substituting Equation (15) in Equations ((11), (13)) and equating like powers of $R$, one obtains the boundary value problem for $j=0$ and $j=1$ as

$$
\frac{\mathrm{d}^{2} u_{0}}{\mathrm{~d} y^{2}}-M^{2} u_{0}=-\left[\theta_{0}+N \phi_{0}\right]
$$




$$
\begin{gathered}
\frac{\mathrm{d}^{2} u_{1}}{\mathrm{~d} y^{2}}-M^{2} u_{1}=-\left[\theta_{1}+N \phi_{1}\right] \\
\frac{\mathrm{d}^{2} \theta_{0}}{\mathrm{~d} y^{2}}=-D_{f}\left[\frac{\mathrm{d}^{2} \phi_{0}}{\mathrm{~d} y^{2}}\right] \\
\frac{\mathrm{d}^{2} \theta_{1}}{\mathrm{~d} y^{2}}=-\left[\frac{4}{3} R\left(C_{T}+\theta_{0}\right)^{3}\right] \frac{\mathrm{d}^{2} \theta_{0}}{\mathrm{~d} y^{2}}-4\left[C_{T}+\theta_{0}\right]^{2}\left(\frac{\mathrm{d} \theta_{0}}{\mathrm{~d} y}\right)-D_{f}\left[\frac{\mathrm{d}^{2} \phi_{1}}{\mathrm{~d} y^{2}}\right] \\
\frac{\mathrm{d}^{2} \phi_{0}}{\mathrm{~d} y^{2}}=-S_{t}\left[\frac{\mathrm{d}^{2} \theta_{0}}{\mathrm{~d} y^{2}}\right] \\
\frac{\mathrm{d}^{2} \phi_{1}}{\mathrm{~d} y^{2}}=-S_{t}\left[\frac{\mathrm{d}^{2} \theta_{1}}{\mathrm{~d} y^{2}}\right]
\end{gathered}
$$

The relevant boundary conditions to be satisfied are:

$$
\begin{aligned}
& u_{0}=u_{1}=\theta_{1}=\phi_{1}=0 ; \phi_{0}=\phi_{0}=1 \quad \text { at } y=0 \\
& \left.u_{0}=u_{1}=\theta_{1}=\phi_{1}=0=\phi_{0}=\phi_{0}=1 \text { at } y=1\right\}
\end{aligned}
$$

The required solutions of the governing steady state energy, concentration and momentum Equations ((16)-(21)) subject to boundary condition (22) are:

$$
\begin{gathered}
\theta(y)=[1-y]+R \chi_{1}\left[2 B^{2}\left(y-y^{2}\right)-\frac{4}{3} B\left(y-y^{3}\right)+\frac{1}{3}\left(y-y^{4}\right)\right] \\
\phi(y)=[1-y]+R S_{t} \chi_{1}\left[2 B^{2}\left(y^{2}-1\right)-\frac{4}{3} B\left(y-y^{3}\right)+\frac{4}{3}\left(y-y^{4}\right)\right] \\
u(y)=\left[\frac{1+N}{M^{2}}\right]\{(1-y)+\sinh (M y-M)\}-R\left\{K_{0} \frac{\sinh (M-M y)}{\sinh (M)}\right. \\
+K_{0}\left(1-\frac{\sinh (M y)}{\sinh (M)}\right)+K_{1}\left(y-\frac{\sinh (M y)}{\sinh (M)}\right)+K_{1}\left(y^{2}-\frac{\sinh (M y)}{\sinh (M)}\right) \\
\left.+K_{2}\left(y^{2}-\frac{\sinh (M y)}{\sinh (M)}\right)+K_{3}\left(y^{3}-\frac{\sinh (M y)}{\sinh (M)}\right)+K_{4}\left(y^{4}-\frac{\sinh (M y)}{\sinh (M)}\right)\right\}
\end{gathered}
$$

Using (23), we write the steady state rate of heat (Nusselt number) transfer on the boundary:

$$
N u_{0}=-\left.\frac{\mathrm{d} \theta}{\mathrm{d} y}\right|_{y=0}=1-R\left[2 B^{2}-\frac{4}{3} B+\frac{1}{3}\right]\left[1+\left(1+C_{T}\right)^{3}\right]
$$

The steady state rate of mass (Sherwood number) transfer on the boundary plate from (24) as:

$$
S h_{0}=-\left.\frac{\mathrm{d} \phi}{\mathrm{d} y}\right|_{y=0}=1-R S_{t} \chi_{1}\left[\frac{4}{3}-\frac{4}{3} B\right]
$$

Also, using (25), we write the skin friction coefficient as: 


$$
\begin{aligned}
\tau_{0}= & -\left.\frac{\mathrm{d} u}{\mathrm{~d} y}\right|_{y=0}=\left[\frac{1+N}{M^{2}}\right]\{1+M \cosh (M)\} \\
& -R\left\{K_{1}\left(1-\frac{M \cosh (M)}{\sinh (M)}\right)-\frac{M \cosh (M)}{\sinh (M)} \times\left(K_{2}+K_{3}+K_{4}\right)\right\}
\end{aligned}
$$

where $B=1+C_{T}$ and the constant $K_{i^{\prime} s}$ and $\chi_{i^{\prime} s}$ are remove in order to reduce the size of the work.

\section{Numerical Procedure}

The nonlinear partial differential Equations ((7)-(9)) are solved numerically using semi-implicit finite difference scheme. We used forward difference formulas for all time derivatives and approximate both the second and first spatial derivatives with second order central differences. The semi implicit finite difference equation corresponding to Equations ((7)-(9)) is as follows:

$$
\begin{gathered}
\frac{u_{i}^{(k+1)}-u_{i}^{k}}{\delta t}=\frac{u_{i-1}^{(k+1)}-2 u_{i}^{(k+1)}+u_{i+1}^{(k+1)}}{(\delta y)^{2}}-M u_{i}^{(k)}+\left[\theta_{i}^{k}+N \phi_{i}^{(k)}\right] \\
\operatorname{Pr} \frac{\theta_{i}^{(k+1)}-\theta_{i}^{k}}{\delta t}=\left[1+\frac{4}{3} R\left(C_{T}+\theta_{i}^{k}\right)^{3}\right]\left(\frac{\theta_{i-1}^{(k+1)}-2 \theta_{i}^{(k+1)}+\theta_{i+1}^{(k+1)}}{(\delta y)^{2}}\right) \\
+4 R\left[C_{T}+\theta_{i}^{(k)}\right]^{2}\left(\frac{\theta_{i+1}^{(k)}-\theta_{i-1}^{(k)}}{2(\delta y)}\right)^{2}+D_{f}\left(\frac{\phi_{i-1}^{(k)}-2 \phi_{i}^{(k)}+\phi_{i+1}^{(k)}}{(\delta y)^{2}}\right) \\
\operatorname{SC} \frac{\phi_{i}^{(k+1)}-\phi_{i}^{k}}{\delta t}=\left(\frac{\phi_{i-1}^{(k+1)}-2 \phi_{i}^{(k+1)}+\phi_{i+1}^{(k+1)}}{(\delta y)^{2}}\right)
\end{gathered}
$$

Using the known values of $\theta$ and $\phi$ at grid point $t=0$ and reducing the solution to tri-diagonal matrix we obtained temperature and concentration fields at time $t_{i+1}=t_{i}+\delta t$ using the known values of the previous time $t=t_{i}$ for all $i=1,2, \cdots, M-1$. Then the velocity field is evaluated using the already known value of temperature and concentration fields obtained at $t_{i+1}=t_{i}+\delta t$. These processes are repeated till the required solution of $\theta, \phi$ and $u$ are gained at convergence criteria.

$$
\left|(u, \theta, \phi)_{\text {exact }}-(u, \theta, \phi)_{\text {num }}\right|<10^{-4}
$$

The iterative system does not restrict time step and the technique is always convergent and unconditionally stable.

\section{Results and Discussion}

This paper, examined the transient natural convective flow through a vertical channel in the presence of a thermal diffusion, diffusion-thermo and radiative heat transfer under the influence of a uniform magnetic field. The system of governing Equations (7)(9) with the boundary conditions (10) is solved employing the finite difference method. The effects of the flow governing parameters on velocity, temperature, skin friction, Nusselt number and Sherwood number, have been shown using line graph as demon- 
strated in Figure 2 through Figure 25. These result show the variations in the velocity field, temperature distribution, skin friction, and heat transfer rate, influenced by the material parameters of the flow problem, that is, the magnetic parameter $(M)$, radiation parameter $(R)$, temperature difference parameter $\left(C_{T}\right)$, sustention parameter $(N)$, dimensionless time $(t)$, Prandtl number $(\mathrm{Pr})$, Schmidt number $(S c)$, thermal diffusion parameter $S_{t}$ and diffusion-thermo parameter $\left(D_{f}\right)$. The radiation parameter $R$ is in the range of $0 \leq R \leq 1$ because terms associated with $R$ behave as strong heat sources and large value of $R$ leads to finite time temperature blow up (see [15]). Prandtl number 0.71, 1.0, 3.0 and 7.0, which physically represent air, electrolyte solution, saturated liquid freon and water respectively. In air the diffusing chemical species of common interest have Schmidt number 0.1 1.0 [16]: the work consider $\mathrm{NH}_{3}$ for $S c=0.78, \mathrm{H}$ for $S c=0.22$ and $\mathrm{H}_{2} \mathrm{O}$ for $S c=0.60$ respectively. Moreover time is selected in the range $0.02 \leq t \leq 1$, in order to capture the steady state situation for air and water. Besides all other parameter is choosing arbitrary.

Figures 2-4 display the velocity, temperature and concentration profiles for different values of dimensionless time $t$ when other controlling parameters are fixed. It is observed that velocity, temperature as well as concentration increases as dimensionless time $t$ increases and attains steady state.

Figure 5 indicates that high values of sustention parameter substantially increase the velocity.

Figures 6-8 depict the effect of Prandtl number on velocity, temperature and concentration. It is seen that an increase in the values of $\operatorname{Pr}$ leads to the decrease in fluid velocity, temperature and concentration. This may be attributed to the fact that at higher Prandtl number, convection currents become weak and qualitatively decreases the temperature, which leads to decrease in velocity and concentration.

Figure 9 illustrates the effect of magnetic $M$ parameter on velocity. It is seen that an increase in the values of leads to the decrease in fluid velocity. This is true since magnetic parameter produce resistive force, which acts opposite direction to the fluid

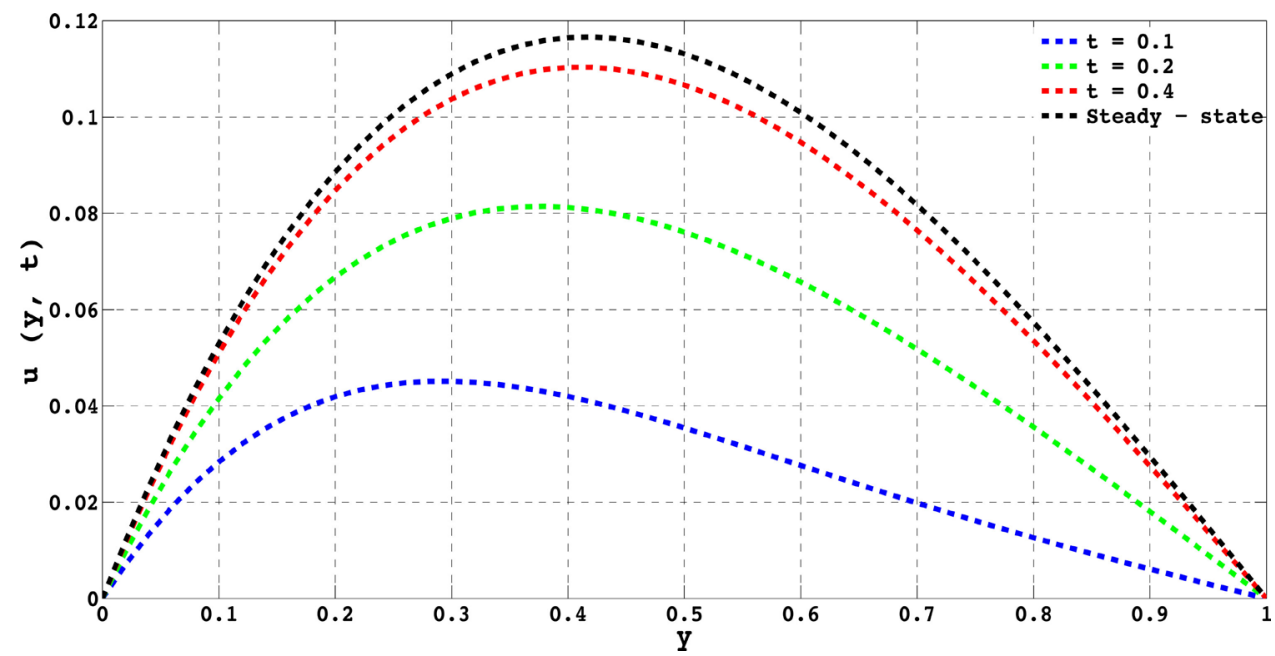

Figure 2. Velocity profile when $C_{T}=0.01, \operatorname{Pr}=0.71, R=0.001, S c=0.22$ and $S_{t}=0.02$. 


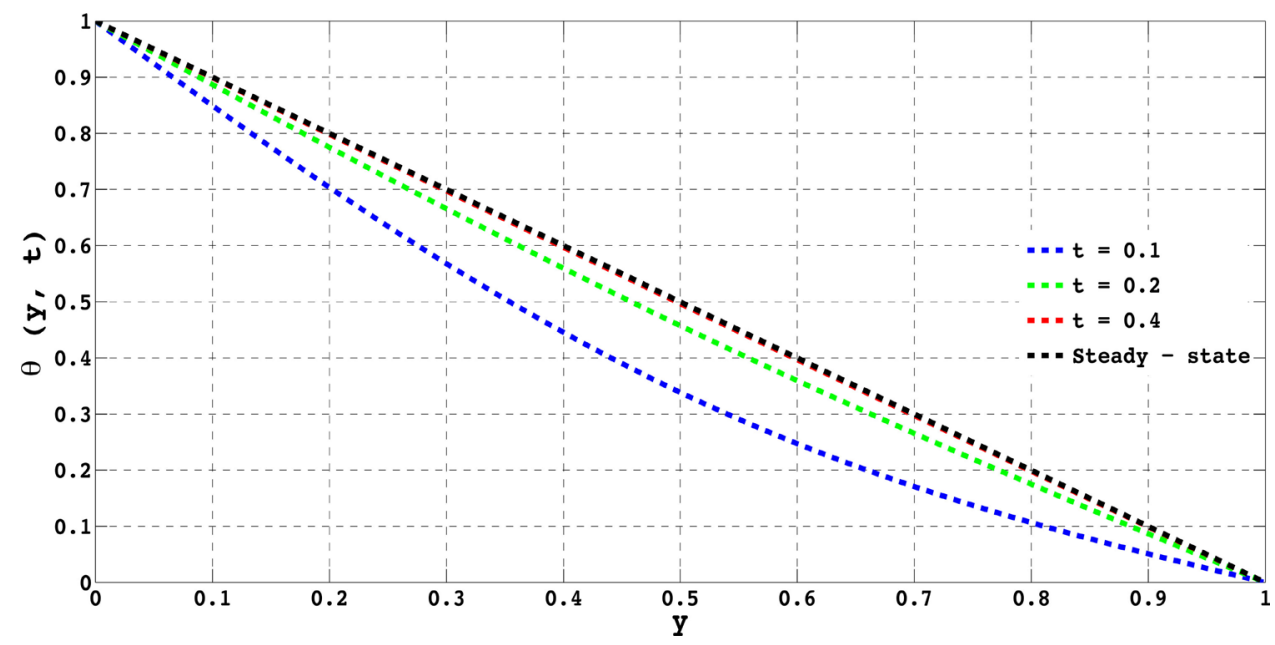

Figure 3. Temperature profile when $C_{T}=0.01, D_{f}=0.02, N=1 \operatorname{Pr}=0.71, R=0.001$, Sc $=0.78$ and $S_{t}=0.02$.

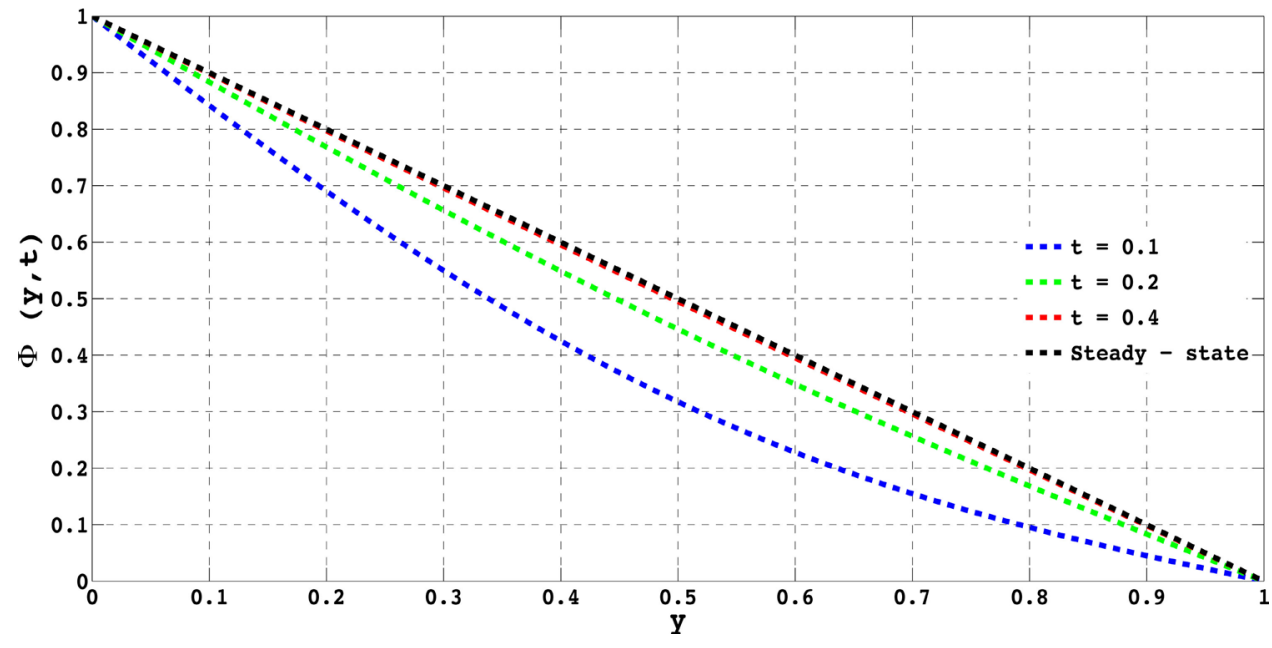

Figure 4. Concentration profile when $D_{f}=0.02, N=1, \operatorname{Pr}=0.71, R=0.001$ and $S c=0.78$.

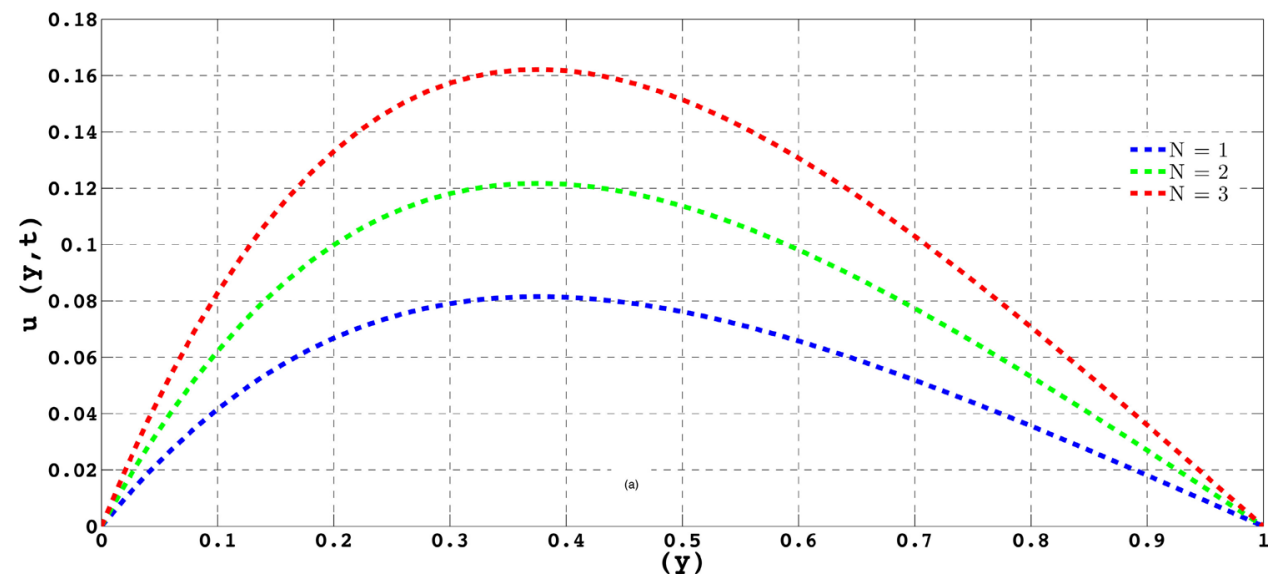

Figure 5. Velocity profile when $C_{T}=0.01, D_{f}=0.02, \quad M=1, \operatorname{Pr}=0.71, R=0.001$, Sc $=0.22, S_{t}=0.02$ and $t=0.2$. 


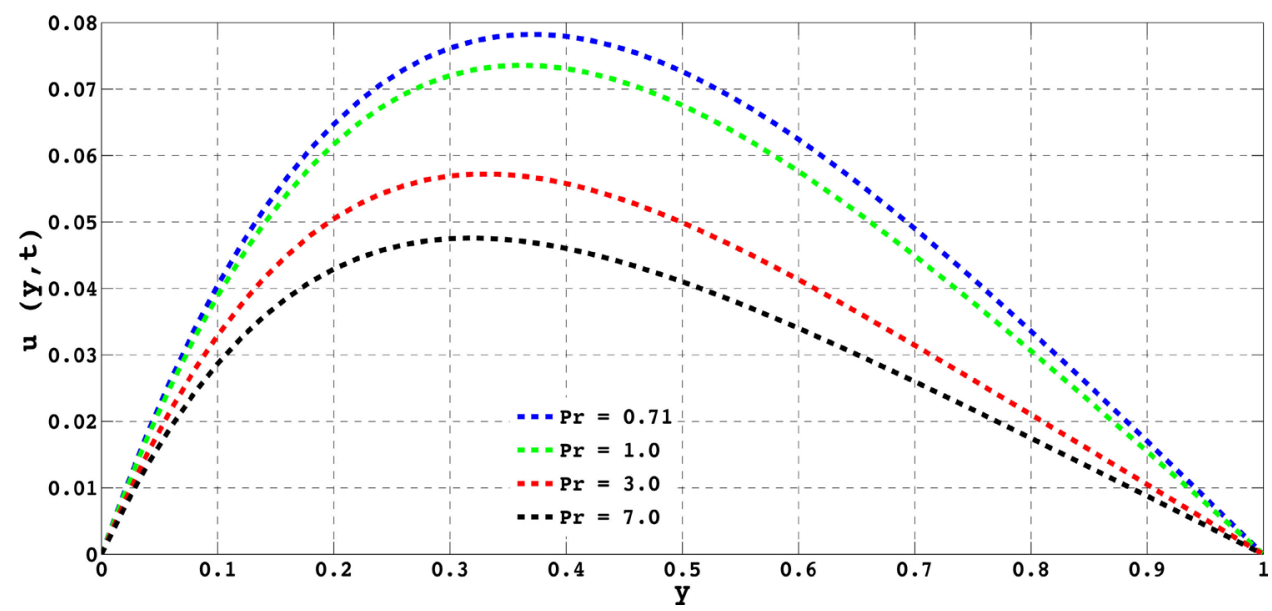

Figure 6. Velocity profile when $C_{T}=0.01, D_{f}=0.02, \quad M=1, \quad N=1, \quad R=0.001$, $S C=0.22, S_{t}=0.02$ and $t=0.2$.

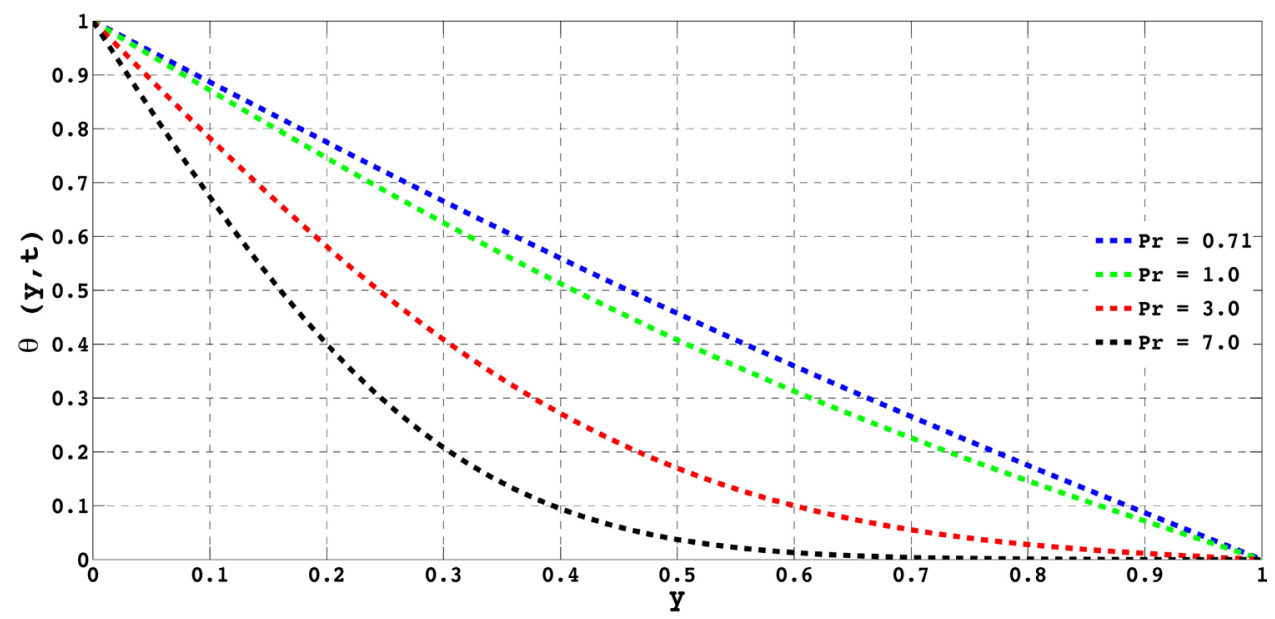

Figure 7. Temperature profile when $C_{T}=0.01, D_{f}=0.02, N=1, R=0.001, S c=0.78$ and $t=0.2$.

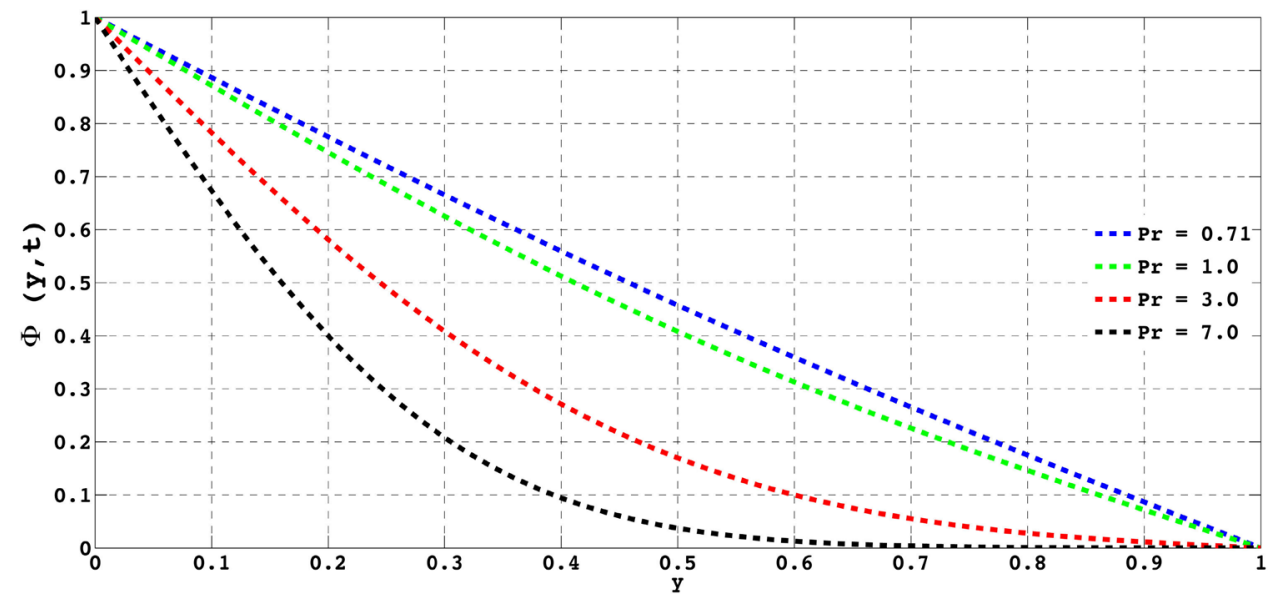

Figure 8. Concentration profile when $D_{t}=0.02, N=1, R=0.001, S c=0.78, S_{t}=0.02$ and $t=0.2$. 


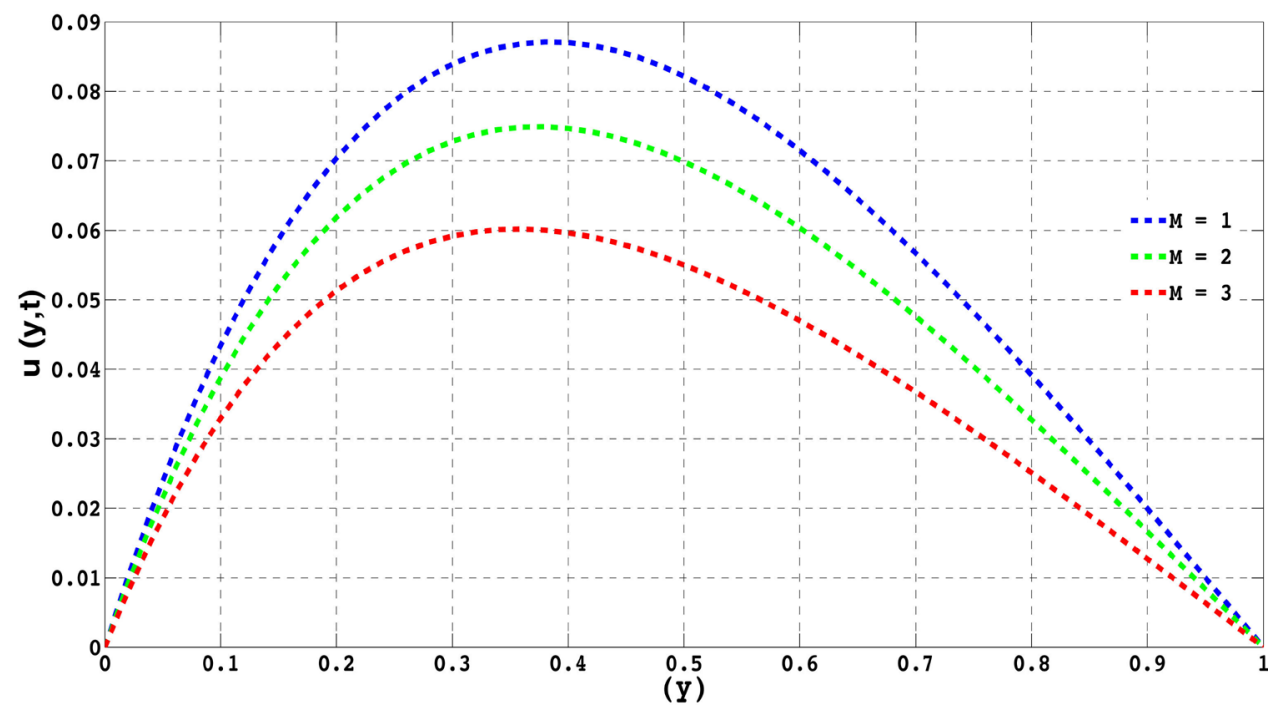

Figure 9. Velocity profile when $C_{T}=0.01, D_{f}=0.02, N=1, \operatorname{Pr}=0.71, \quad R=0.001$, Sc $=0.22, S_{t}=0.02$ and $t=0.2$.

motion. This is an important controlling mechanism inflow and heat transfer processes so that the finished product meet the desire quality specification.

Figure 10 show the influence of Schmidt Sc number on velocity. The velocity is observed to descend with Schmidt number when other numerical values are treated constant.

Figure 11 and Figure 12 illustrate the disparity in both velocity and temperature due to a change in thermal-diffusion parameter $D_{f}$ when other parameters are fixed. From these Figures it is noted that the effect of $D_{f}$ is to increase both velocity and temperature respectively.

Figures 13-15 explain the effect of increasing diffusion-thermo parameter. With all other parameters constant, the velocity, temperature and concentration increases with increase in $S_{t}$.

Figure 16 and Figure 17 illustrate the effect of temperature difference parameter $C_{T}$ on velocity and temperature profiles, respectively. It is observed that as the value of $C_{T}$ increases there is corresponding increase in the temperature of the fluid, and hence cause increase in velocity.

Figure 18 and Figure 19 inspect the influence of the radiation parameter $R$ on the velocity and temperature, respectively. It is observed that the impact of $R$ has insignificant effect on both velocity and temperature.

Figures 20-22 present variation of skin friction at $y=0$ for different values of $R$, $D_{f}, S_{t}$ and $t$. The results show that the values of skin friction increases with increase of $R, D_{f}$ and $S_{t}$ respectively. In addition the result show that skin friction increases with dimensionless time $t$ until steady state is attain.

Figures 23-25 present the variation of rate of heat transfer for different values of $R$, $D_{f}, S_{t}$ and $t$ at $y=0$. It is observed in Figure 23 that the rate of heat transfer increases with increase in $R$. The physical fact is that an increase in radiation adds more 


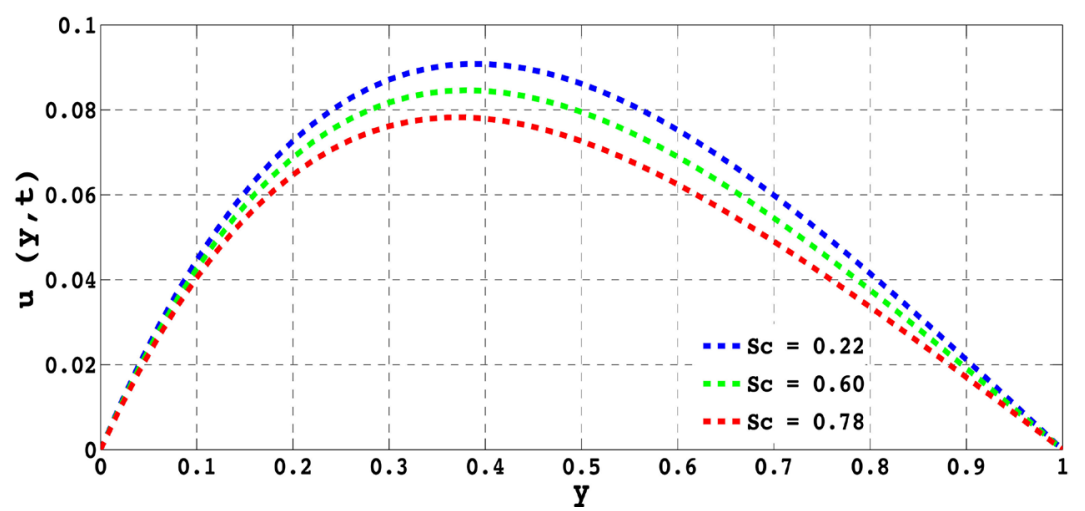

Figure 10. Velocity profile when $C_{T}=0.01, \quad D_{f}=0.02, \quad M=1, \quad N=1, \operatorname{Pr}=0.71$ $R=0.001, S_{t}=0.02$ and $t=0.2$.

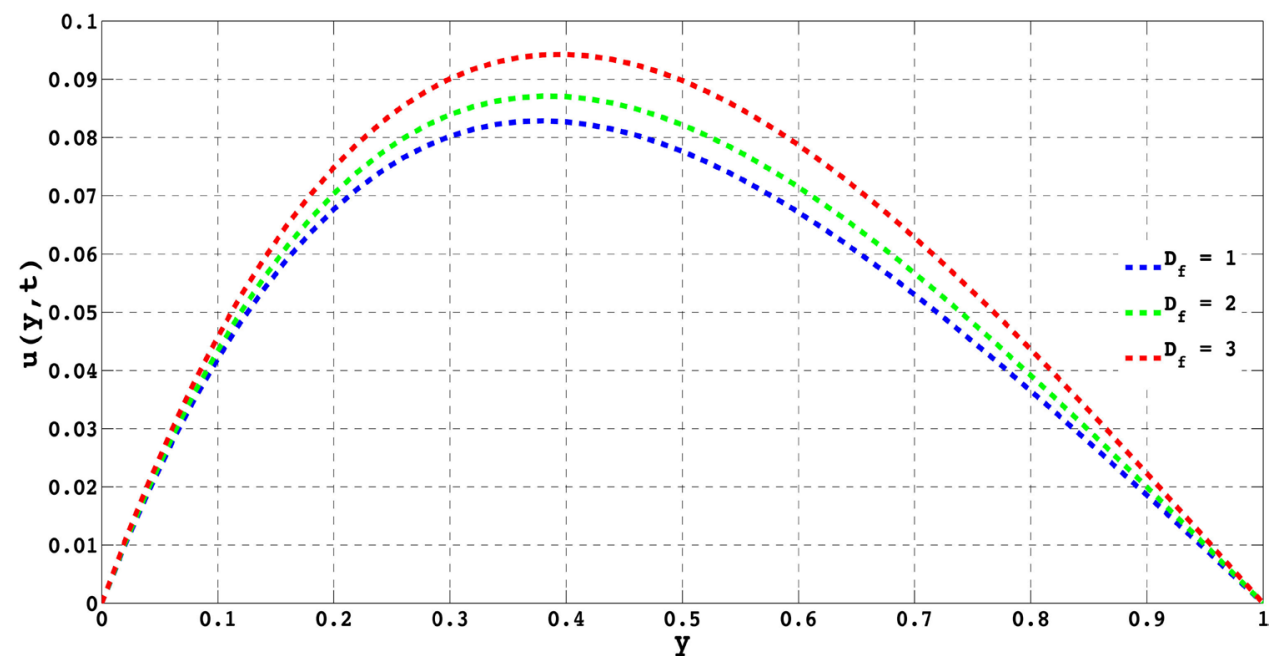

Figure 11. Velocity profile when $C_{T}=0.01, \quad M=1, \quad N=1, \operatorname{Pr}=0.71, \quad R=0.001$, Sc $=0.78, S_{t}=0.02$ and $t=0.2$.

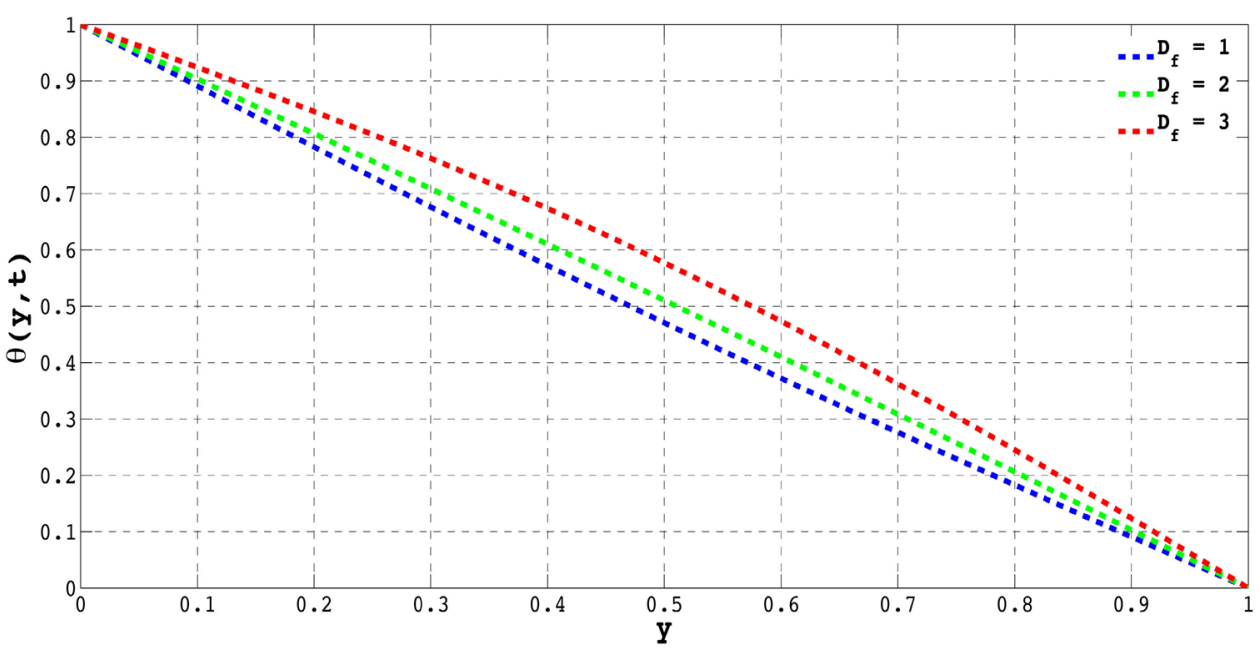

Figure 12. Temperature profile when $C_{T}=0.01, N=1, \operatorname{Pr}=0.71, R=0.001, S c=0.78$ and $t=0.2$. 


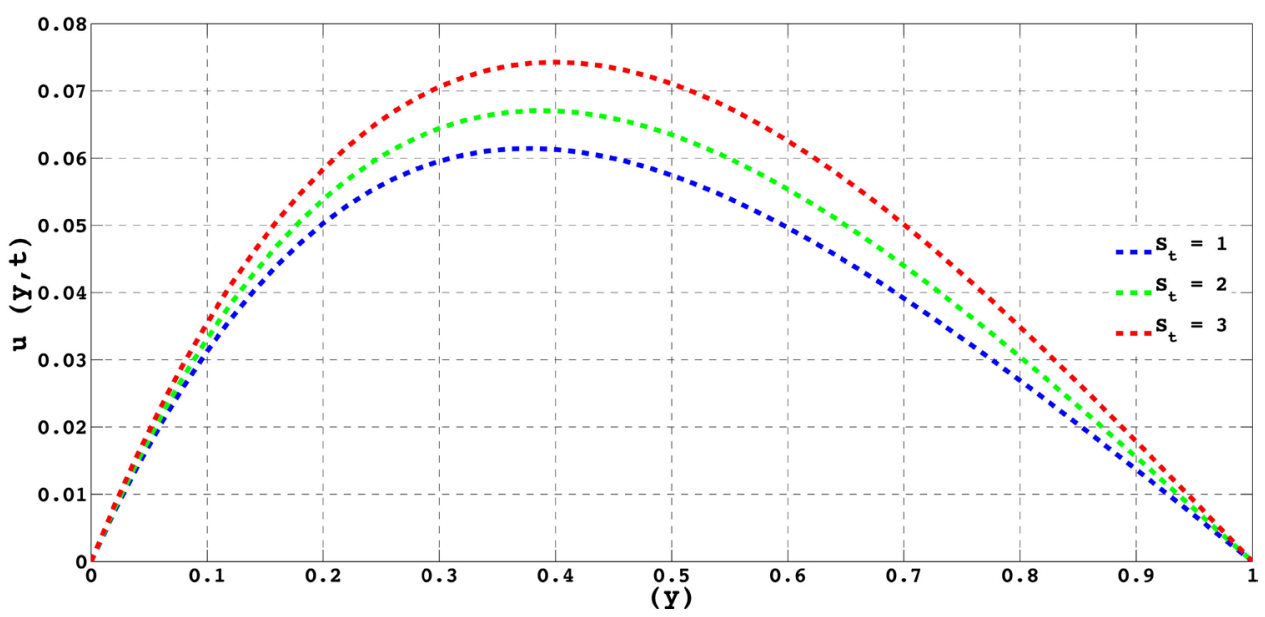

Figure 13. Velocity profile when $C_{T}=0.01, D_{f}=0.02, \quad M=1, \quad N=1, \operatorname{Pr}=0.71$, $R=0.001, S c=0.78$, and $t=0.2$.

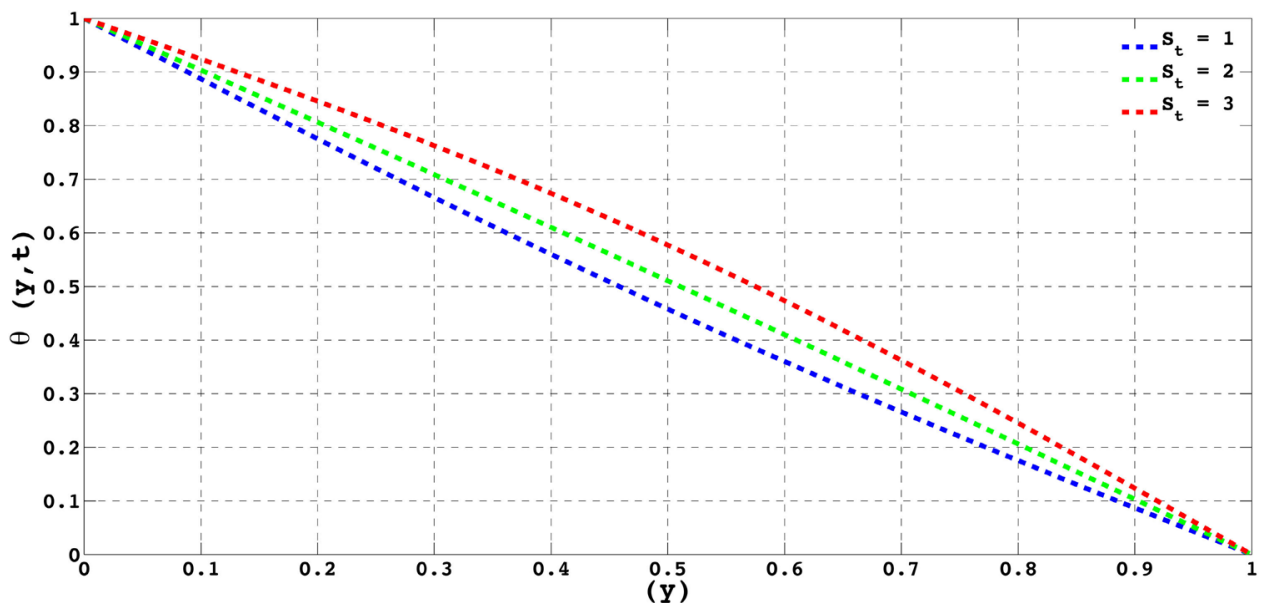

Figure 14. Temperature profile when $C_{T}=0.01, N=1, \operatorname{Pr}=0.71, R=0.001, S c=0.78$ and $t=0.2$.

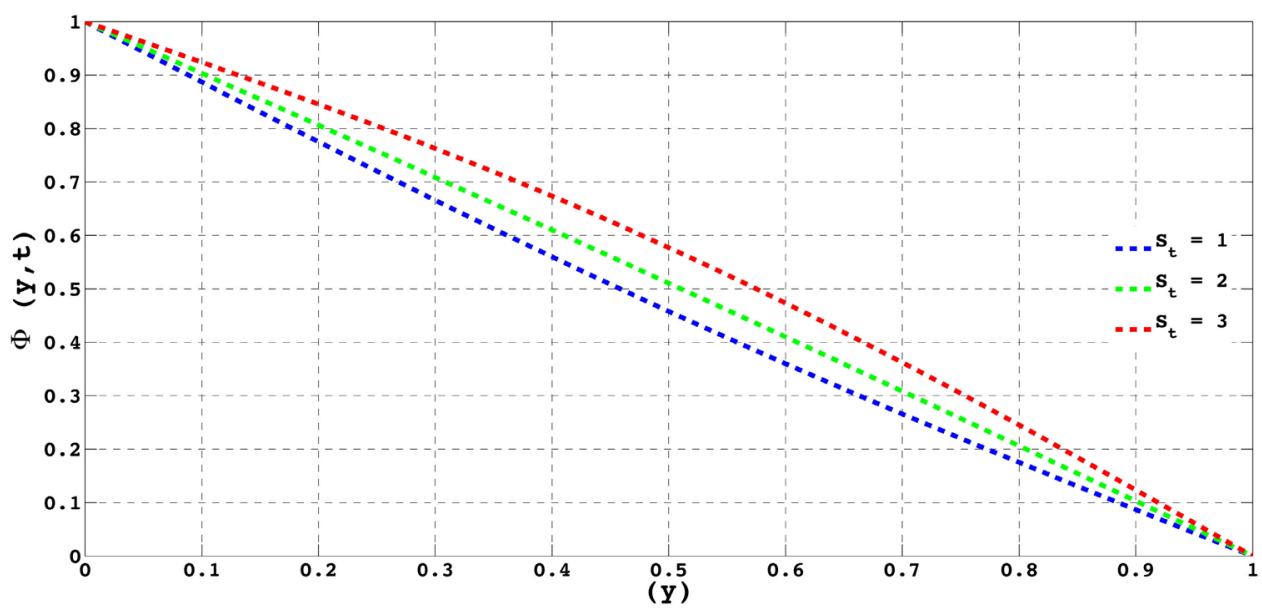

Figure 15. Concentration profile when $D_{t}=0.02, \quad N=1, \operatorname{Pr}=0.71, \quad R=0.001, \quad S c=0.078$ and $t=0.2$. 


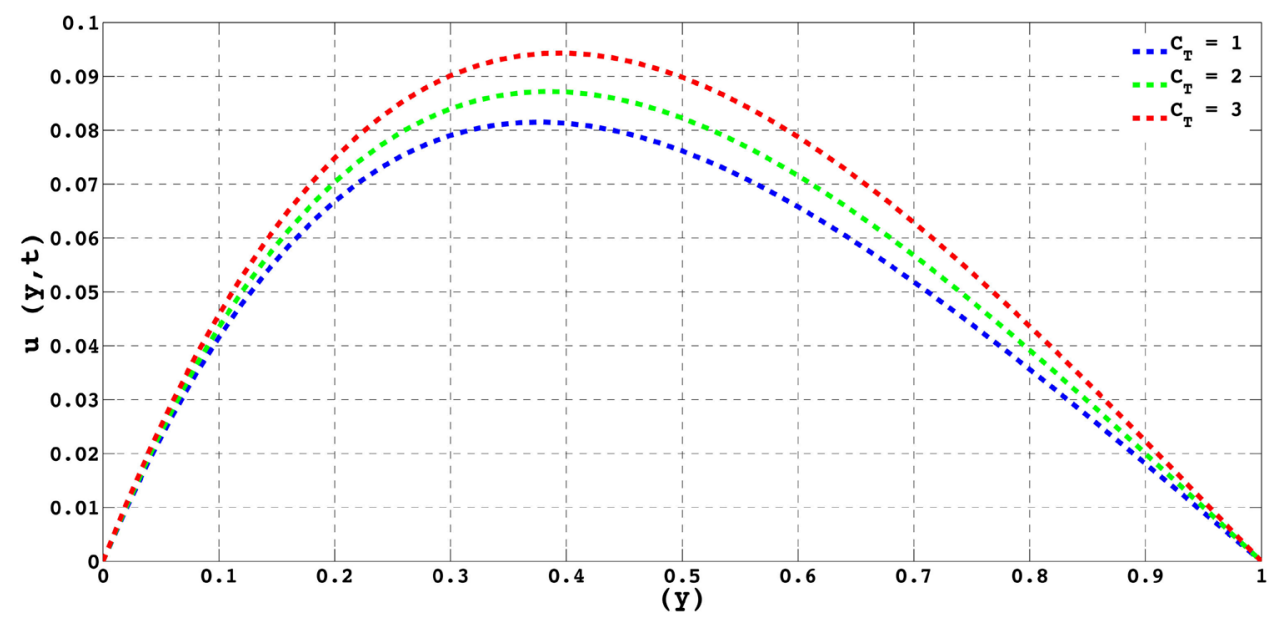

Figure 16. Velocity profile when $D_{t}=0.02, \quad M=1, N=1, \operatorname{Pr}=0.71, R=0.001$, Sc $=0.78, S_{t}=0.02$ and $t=0.2$.

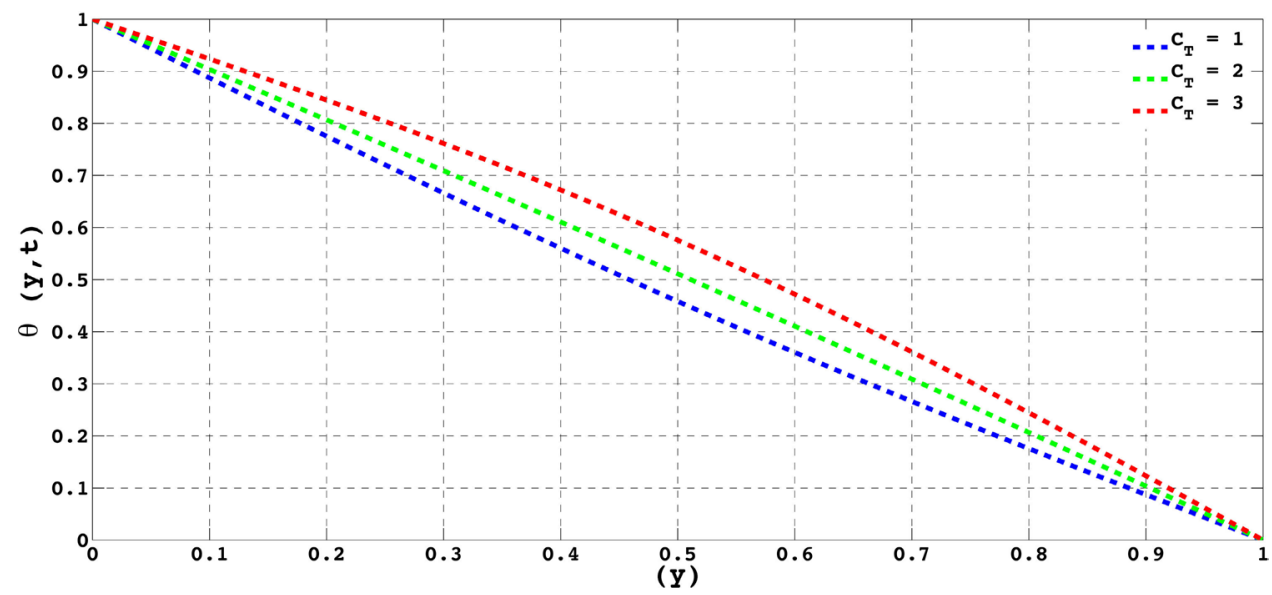

Figure 17. Temperature profile when $D_{t}=0.02, N=1, \operatorname{Pr}=0.71, R=0.001, S c=0.78$ and $t=0.2$.

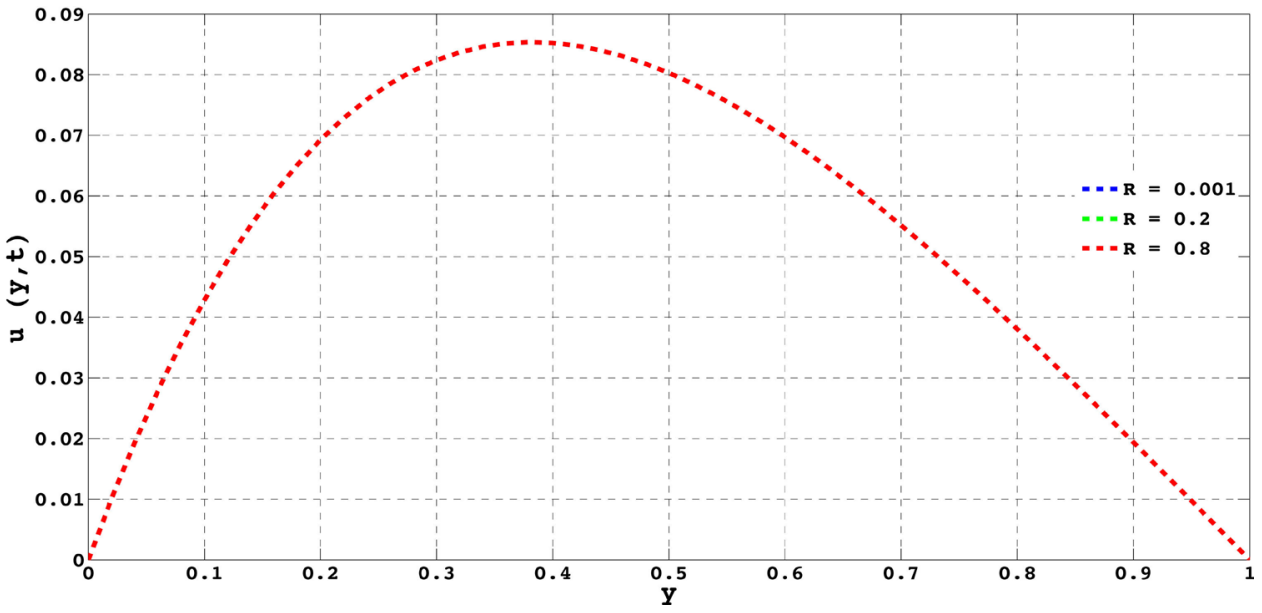

Figure 18. Velocity profile when $C_{T}, D_{f}=0.02, M=1, N=1, \operatorname{Pr}=0.71, R=0.001$, Sc $=0.78, S_{t}=0.02$ and $t=0.2$. 


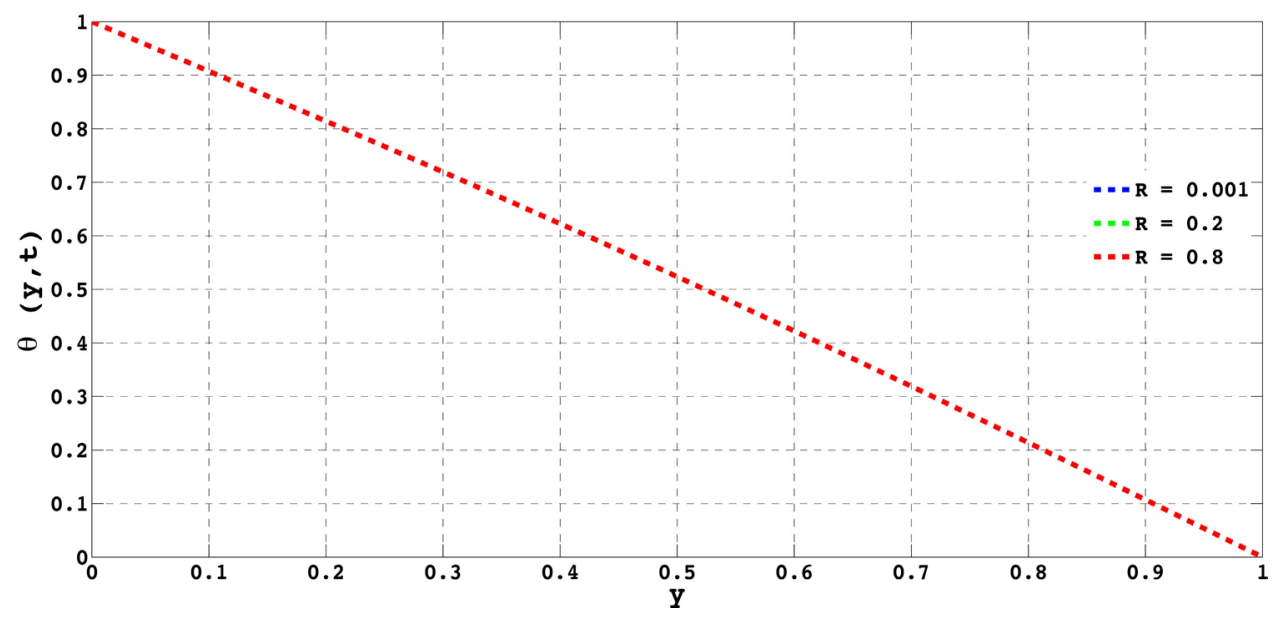

Figure 19. Temperature profile when $C_{T}=0.01, D_{t}=0.02, N=1, \operatorname{Pr}=0.71, R=0.001$, Sc $=0.78$ and $t=0.2$.

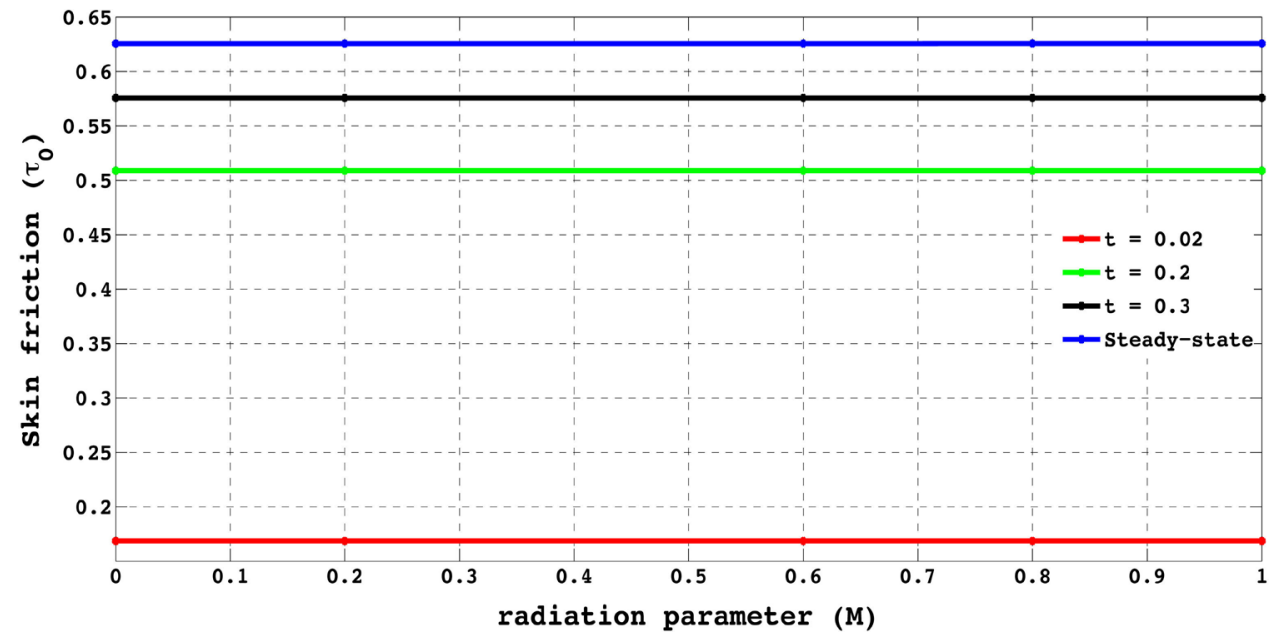

Figure 20. Skin friction against $R$ at $y=0$.

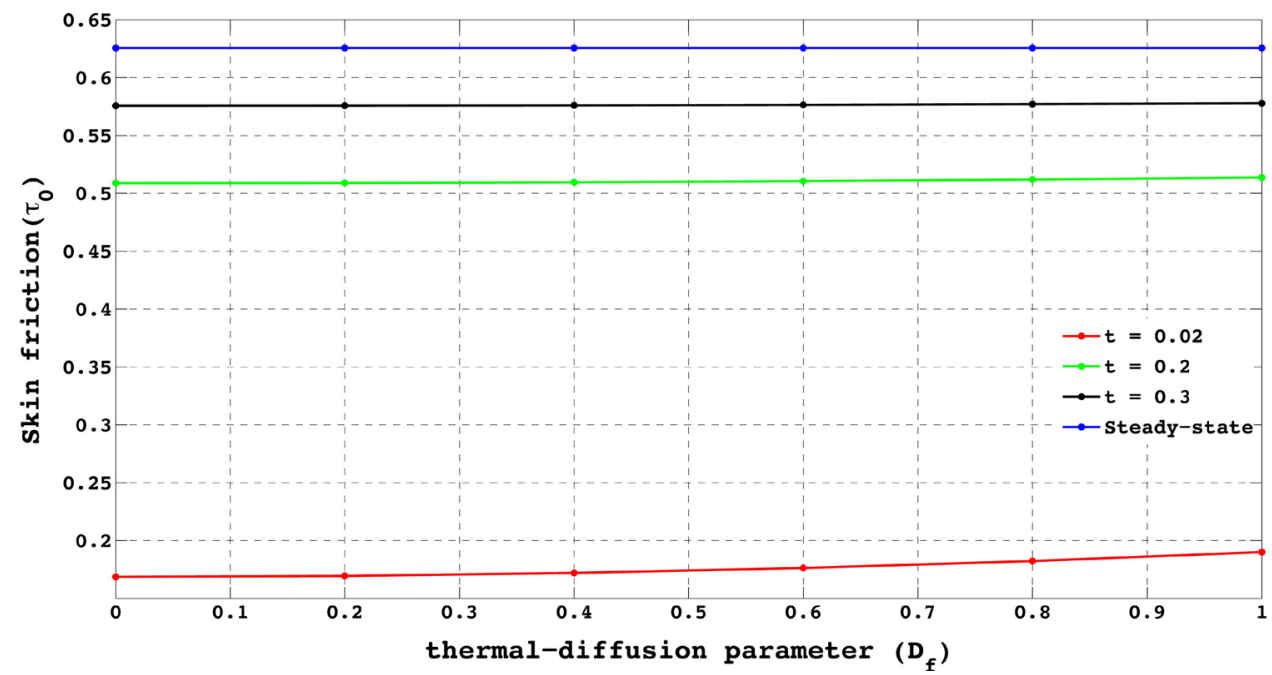

Figure 21. Skin friction against $D_{f}$ at $y=0$. 


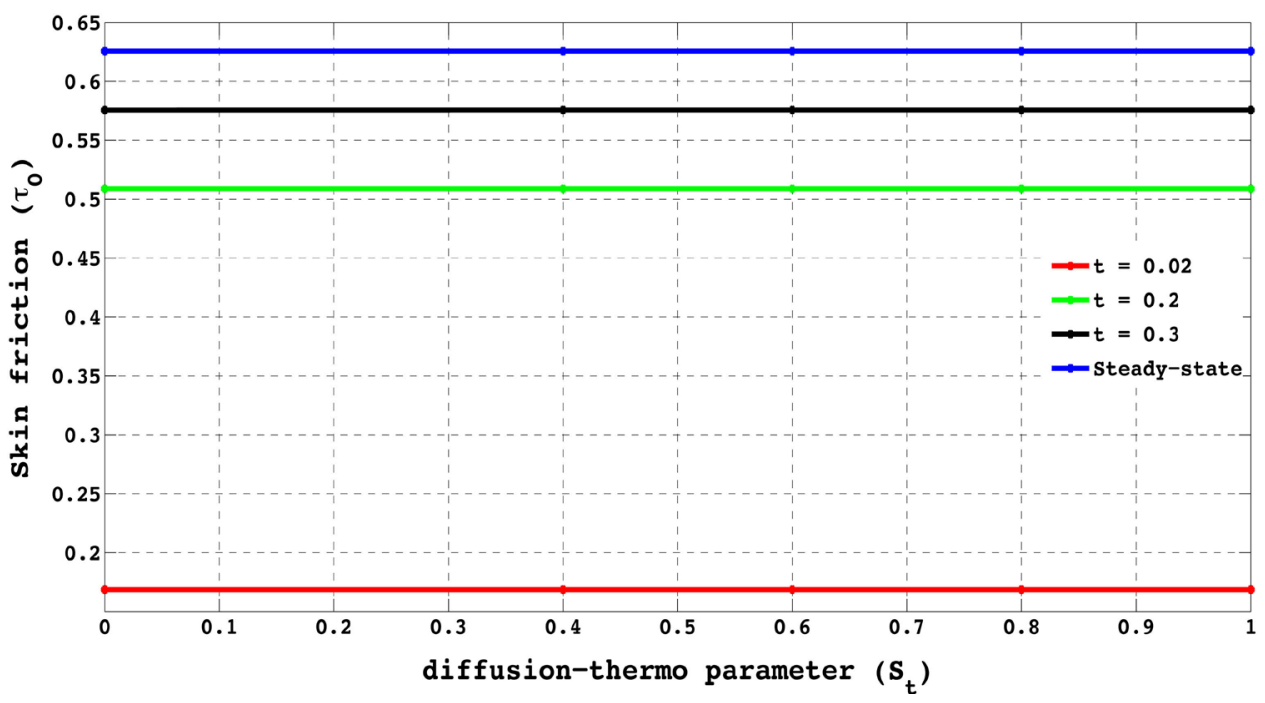

Figure 22. Skin friction against $S_{t}$ at $y=0$.

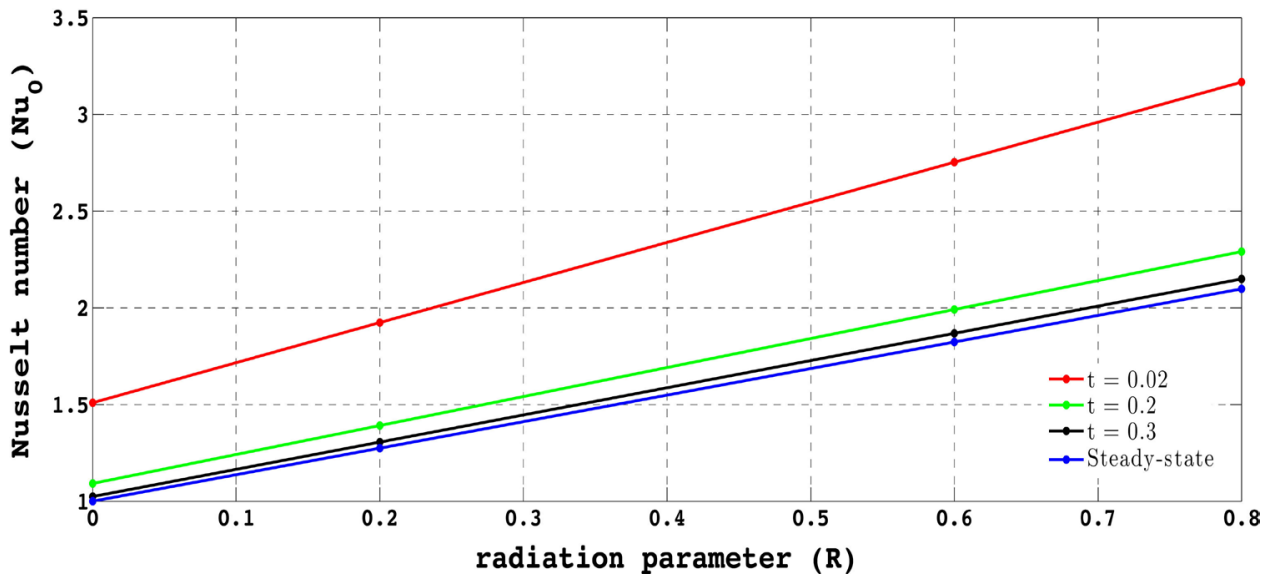

Figure 23. Nusselt number against $R$ at $y=0$.

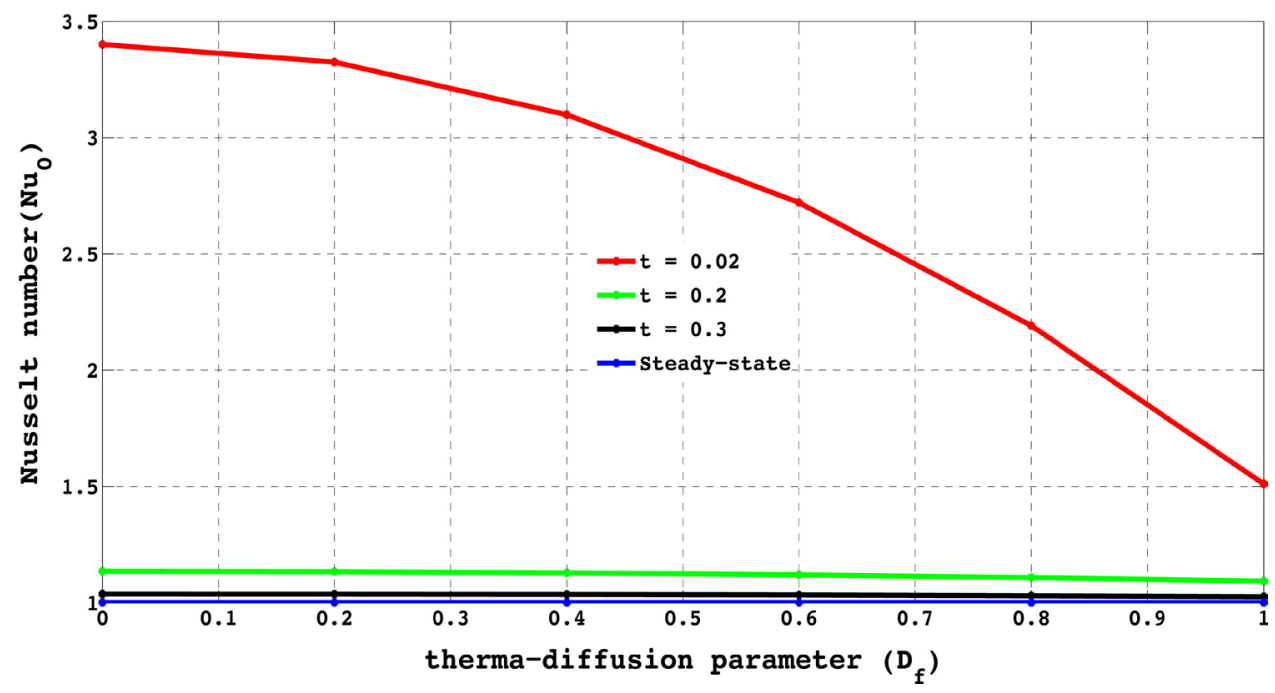

Figure 24. Nusselt number against $D_{f}$ at $y=0$. 


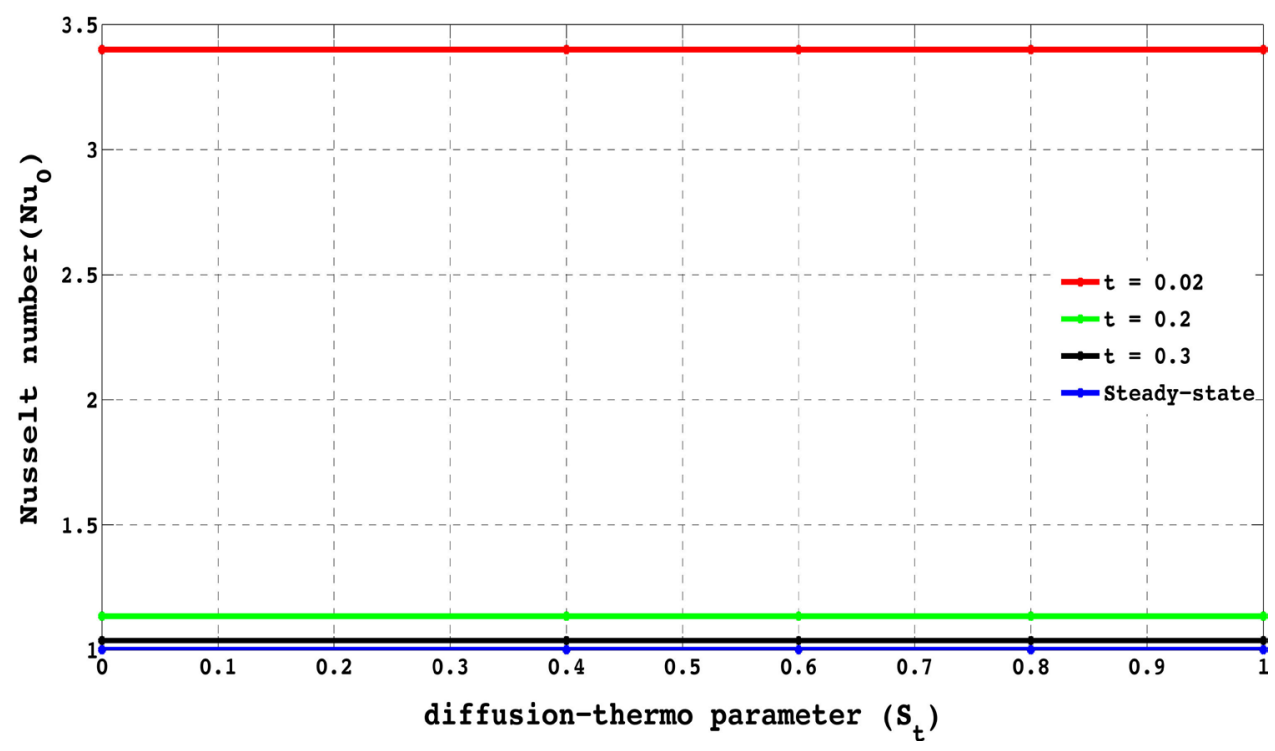

Figure 25. Nusselt number against $S_{t}$ at $y=0$.

heat to the fluid and lead to an increase temperature, which correspond to the increase in rate of heat transfer. Figure 24 and Figure 25 show that the effect of $D_{f}$ and $S_{t}$ has insignificant effect to the values of rate of heat transfer. Furthermore as dimension time increases the rate of heat transfer decreases (see Figures 23-25). This may be attributed that the heat is been driven away from plate $y=0$ through fluid to the other plate $y=1$.

\section{Result Validation}

In order to verify the accuracy of our results, the analytical solutions (steadystate) obtained from Equation (25) by Perturbation method was compared with that of numerical solution derived by semi-implicit finite difference from Equation (29). These computed results are tabulated in Table 1. It is interesting to observe from this Table 1 that the transient and steady state solutions results (under some limiting conditions) are in very good agreement at large value of time, which clearly shows the correctness of our numerical (computed) scheme.

\section{Conclusions}

Transient MHD natural convection flow in a vertical channel in a presence of thermal radiation, thermal diffusion and diffusion-thermo effects confined between two infinite vertical parallel plates has been investigated. The model representing the present physical situation is highly nonlinear due to the presence of thermal radiation effect. The non-linear differential equations under appropriate initial and boundary condition are numerically solved using implicit finite difference method. Also analytical solutions are derived by regular perturbation method for steady state situation. The impact of the essential dimensionless controlling parameter on velocity, temperature, concentration, skin-friction and Nusselt number is presented with the aid of line graphs and discussed. 
Table 1. Comparison between numerical values of transient velocity obtained using finite difference method and steady state velocity obtained by Perturbation method.

\begin{tabular}{lcc}
\hline & Analytical solutions (Perturbation method) & $\begin{array}{c}\text { Numerical solutions at large time } \\
(\mathrm{t}=1.0)\end{array}$ \\
\hline$y$ & $u(y)$ & $u(y, t)$ \\
\hline 0.0 & 0.0 & 0.0 \\
0.1 & 0.052829450187355 & 0.052891525028535 \\
0.2 & 0.088409716341568 & 0.088323183046247 \\
0.3 & 0.108861470010599 & 0.108659409815396 \\
0.4 & 0.116391069074050 & 0.116111597365865 \\
0.5 & 0.113075548985047 & 0.112759421899413 \\
0.6 & 0.100883411538198 & 0.100571136321596 \\
0.7 & 0.081694327474669 & 0.081423079561766 \\
0.8 & 0.057317950114637 & 0.057118614533452 \\
0.9 & 0.029512028310309 & 0.029406666832307 \\
1.0 & 0.0 & 0.0 \\
\hline
\end{tabular}

From the indicated results of the problem, the following observations were concluded:

1) As the radiation, temperature difference, sustention parameter, thermal-diffusion, diffusion-thermo, and non-dimensional time parameters increase, both the velocity and temperature increase while velocity decreases with Schmidt number and magnetic parameter.

2) Increasing Prandtl number reduces values of velocity, temperature and concentration.

3) Skin friction, Nusselt and concentration increase with time.

4) The values of Nusselt number are higher in case of radiation and contrast in case of thermal-diffusion and diffusion-thermo at $y=0$.

5) Good agreement between steady state and transient solution was found after some sufficiently large time $t$.

6) During numerical computation, it is found that the time required reaching steady state velocity and temperature is directly proportional to the Prandtl number of the working fluid. That is the time required to reach steady state velocity and temperature is approximately 0.71 (dimensionless time) for air $(\mathrm{Pr}=0.71)$ for fixed values of other controlling parameters.

\section{Acknowledgements}

We thank the Editor and the referee for their comments. This support is greatly appreciated.

\section{References}

[1] Moreau, R. (1990) Magnetohydrodynamics. Academic Publishers, Dordrecht. https://doi.org/10.1007/978-94-015-7883-7 
[2] Hartman, J. and Lakatos, F. (1937) Kongelige danke videnskabernes selskab. MatematiskFysiske Meddelelser, 15, 6-7.

[3] Makinde, O.D. and Sibanda, P. (2008) Magnetohydrodynamic Mixed Convective Flow and Heat and Mass Transfer past a Vertical Plate in a Porous Medium with Constant Wall Suction. ASME-Journal of Heat Transfer, 130, 11260. https://doi.org/10.1115/1.2955471

[4] Kesavaraya, D.C., Satyanarayana, P.V. and Venkataramana, S.V. (2013) Radiation and Thermo-Diffusion Effects on Mixed Convective Heat and Mass Transfer Flow of a Viscous Dissipated Fluid over a Vertical Surface in the Presence of Chemical Reaction with Heat Source. International Journal of Scientific Engineering and Technology, 2, 56-72.

[5] Eckert, E.R.G. and Dranke, R.M. (1972) Analysis of Heat and Mass Transfer. McGrow-Hill, New York.

[6] Jha, B.K., Samaila, A.K. and Ajibade, A.O. (2011) Diffusion-Thermo Effects on Free Convective Heat and Mass Transfer Flow in a Vertical Channel with Symmetric Boundary Conditions. Journal of Heat Transfer, 133, 1-8. https://doi.org/10.1115/1.4003240

[7] Kumar, R. (2012) Thermo Diffusion and Radiative Heat Effect on Unsteady Oscillatory Mixed Convection MHD Flow through a Planer Channel Filled with Porous Medium. International Journal of Physics and Mathematical Sciences, 3, 13 p.

[8] Ibrahim, F.S.M.E.A. and Bakr, A.A. (2006) Effect of the Chemical Reaction and Radiation Absorption on the Unsteady MHD Free Convection Flow past a Semi Infinite Vertical Permeable Moving Plate with Heat Source and Suction. Communication in Nonlinear Science and Numerical Simulation, 13, 1056-1066.

https://doi.org/10.1016/j.cnsns.2006.09.007

[9] Sudhakar, K., Raju, R.S. and Rangamma, M. (2012) Chemical Reaction Effect on an Unsteady MHD Free Convection Flow past an Infinite Vertical Accelerated Plate with Constant Heat Flux, Thermal Diffusion and Diffusion Thermo. International Journal of Modern and Engineering Research, 2, 3329-3339.

[10] Sharma, B.K., Yazad, K., Mishra, N.K. and Chaudhary, R.C. (2012) Soret and Dufour Effects on Unsteady MHD Mixed Convection Flow past a Radiative Vertical Porous Plate Embedded in a Porous Medium with Chemical Reaction. Applied Mathematics, 3, 717-723. https://doi.org/10.4236/am.2012.37105

[11] Sarada, K. and Shankar, B. (2013) The Effects of Soret and Dufour on an Unsteady MHD Free Convection Flow past a Vertical Porous Plate in the Presence of Suction or Injection. International Journal of Engineering Science, 2, 13-25.

[12] Hayat, T., Awais, M. and Asghar, S. (2013) Radiation Effects in a Three-Dimensional Flow of MHD Eyring-Powell Fluid. Journal of the Egyptian Mathematical Society, 21, 379-384. https://doi.org/10.1016/j.joems.2013.02.009

[13] Ahmed, N., Bhaattacharya, D.J. and Barua, D.P. (2014) Radiation, Soret and Dufour Effects in MHD Channel Flow Bounded by a Long Wavy Wall and a Uniformly Moving Parallel at Wall. Journal of Applied Mathematics and Fluid Mechanics, 6, 1-19.

[14] Ali Agha, H., Bouaziz, M.N. and Hanini, S. (2014) Free Convection Boundary Layer Flow from a Vertical Flat Plate Embedded in a Darcy Medium Filled with a Nanofluid: Effects of Magnetic Field and Thermal Radiation. Arabian Journal for Science and Engineering, 39, 8331-8340. https://doi.org/10.1007/s13369-014-1405-Z

[15] Makinde, O.D. and Chinyoka, T. (2010) Numerical Investigation of Transient Heat Transfer to Hydromagnetic Channel Flow with Radiative Heat and Convective Cooling. Communication in Nonlinear Science and Numerical Simulation, 15, 3919-3930.

https://doi.org/10.1016/j.cnsns.2010.01.013 
[16] Jha, B.K. and Ajibade, A.O. (2009) Free Convective Flow of Heat Generating/Absorbing Fluid between Vertical Porous Plates with Periodic Heat Input. International Communications in Heat and Mass Transfer, 36, 624-631.

https://doi.org/10.1016/j.icheatmasstransfer.2009.03.003

Submit or recommend next manuscript to SCIRP and we will provide best service for you:

Accepting pre-submission inquiries through Email, Facebook, LinkedIn, Twitter, etc. A wide selection of journals (inclusive of 9 subjects, more than 200 journals)

Providing 24-hour high-quality service

User-friendly online submission system

Fair and swift peer-review system

Efficient typesetting and proofreading procedure

Display of the result of downloads and visits, as well as the number of cited articles

Maximum dissemination of your research work

Submit your manuscript at: http://papersubmission.scirp.org/

Or contact am@scirp.org 